\title{
CROWD EVACUATION ANALYSIS OF THE PAPAL BASILICA OF SAINT FRANCIS IN ASSISI, ITALY
}

\author{
FABIO GARZIA ${ }^{1,2}$, MARA LOMBARDI $^{1}$, STEFANO MARSELLA $^{3}$, GIULIANO ROSSI ${ }^{1}$, \\ NICOLÒ SCIARRETTA ${ }^{1}$ \& MASSIMO GUARASCIO ${ }^{1}$ \\ ${ }^{1}$ Safety \& Security Engineering Group-DICMA, SAPIENZA - University of Rome, Rome, Italy. \\ ${ }^{2}$ Foundation for the Basilica of Saint Francis in Assisi, Assisi, Italy. \\ ${ }^{3}$ Italian Department of Fire Brigades, Ministry of Interior, Italy.
}

\begin{abstract}
The safety and security of public events involving large masses, or anyway the safety of crowded sites, is a timely and important topic that must be properly and deeply analysed. It is therefore necessary to use proper methods of analysis and design of the safety and security of public events by a suitable engineering approach, aimed at guaranteeing the safety and security of people during evacuation in ordinary and emergency conditions.

The present work analyses the crowd evacuation of the Papal Basilica of Saint Francis in Assisi that, together with the Sacred Convent, represent a unique and specific cultural heritage site where the mortal remains of St. Francis have been housed since 1230 AD. Millions of pilgrims and visitors from all over the world visit this site each year. In 2000 AD, together with other Franciscan sites in the surrounding area, it achieved UNESCO World Heritage status.

To reach the desired goal, a proper simulation model of people evacuation was set up, considering the fundamental aspects of interaction between the architecture and layout of the Basilica and the behaviour of the crowd.

The obtained results provide a series of important information for people evacuation management that leads to a more regular exit flow with a decrease in the time of stationary waiting and, therefore, with a decrease of the probability of generating spontaneous panic phenomena induced by the crowd. Keywords: crowd evacuation, emergency management, safety for evacuation, security for evacuation.
\end{abstract}

\section{INTRODUCTION}

Public events are an increasingly common feature of modern society: every year in the communities of the whole world there are many events of any kind such as sports shows, ceremonies, fairs, etc.

The growing population density together with the greater ease of moving from one geographic area to another brings together a great number of people at mass events such as rock concerts, sporting or religious events, etc.

The mere fact that hundreds or thousands of people gather in the same place and at the same time makes these events more dangerous than one would expect. In fact, unfortunately, very often accidents have happened on such occasions that have caused the loss of human lives.

It is therefore necessary to carry out a specific risk assessment that considers all the characteristics of each event, in order to guarantee an adequate level of safety and security for all people who participate in them in any way.

It is also necessary to avoid considering that mass events (or anyway normally crowded sites such as the one considered in the present work and illustrated in the following) of the same type are characterized by the same factors and therefore by the same level of risk. The kind of event represents only the starting point necessary for an ad hoc risk assessment that can effectively support qualified personnel who must manage it to guarantee a suitable level of safety and security, maybe aided by suitable integrated technological system, even based 
on IoT (Internet of Things) / IoE (Internet of Everything) capable of supporting a proper multidisciplinary model for safety and security management [1-5].

Due to the actual worldwide situation, people begin to be afraid of gathering in a large crowd and for this reason it is recommended to reassure them in a proper way. Furthermore, it is necessary to remind that to generate panic among the crowd any threat is enough, even if only presumed, like a scream or a loud and sudden noise [6-13].

The safety and security of public events involving large masses, or anyway the safety of crowded sites, is therefore a timely and important topic that must be properly and deeply analysed. It is therefore necessary to use proper methods of analysis and design of the safety and security of public events by a suitable engineering approach, aimed at guaranteeing the safety and security of people during evacuation in ordinary and emergency conditions.

The present crowd evacuation analysis is focused on the Papal Basilica of Saint Francis in Assisi that, together with the Sacred Convent, represent a unique and specific cultural heritage site visited by millions of pilgrims and visitors from all over the world each year.

To reach the desired goal, a proper simulation model of people evacuation was set up, considering the fundamental aspects of interaction between the architecture and layout of the Basilica and the behaviour of the crowd.

The obtained results provide a series of important information for people evacuation management that leads to a more regular exit flow with a decrease in the time of stationary waiting and, therefore, with a decrease in the probability of generating spontaneous panic phenomena induced by the crowd.

\section{THE PAPAL BASILICA AND SACRED CONVENT OF SAINT FRANCIS IN ASSISI}

The Papal Basilica of Saint Francis in Assisi, together with the Sacred Convent, represents a unique and specific cultural heritage site where the mortal remains of St. Francis have been housed since $1230 \mathrm{AD}$. Millions of pilgrims and visitors from all over the world visit this site each year. The considered site is shown in Fig. 1.

In $2000 \mathrm{AD}$, together with other Franciscan sites in the surrounding area, it achieved UNESCO World Heritage status. Important international events, such as those related to world peace and dialogue between religions, are organized at this site and are often attended by thousands of people. The Papal Basilica, where unique frescos by Giotto and other famous painters are displayed, comprises three stratified structures: the tomb of St. Francis, located at the lower level; the lower Church, whose altar is just above the tomb of St. Francis; and the upper Church, located above the lower Church. Inside the Sacred Convent there is a museum,

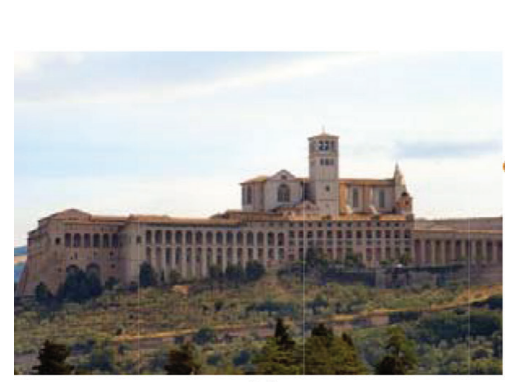

(a)

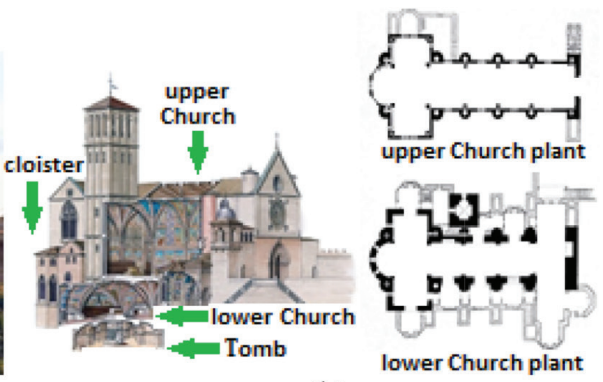

(b)

Figure 1: (a) Panoramic view of the considered site; (b) view of the 3 layers of the Basilica. 


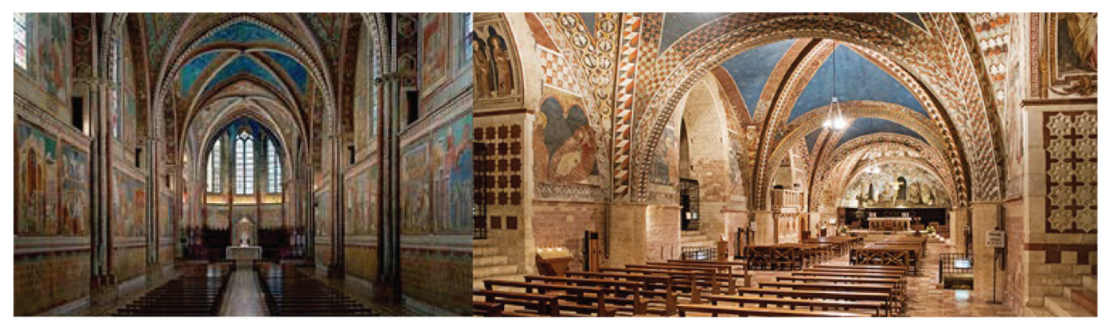

(a)

(b)

Figure 2: View of the interior of: (a) upper Church; (b) lower Church.

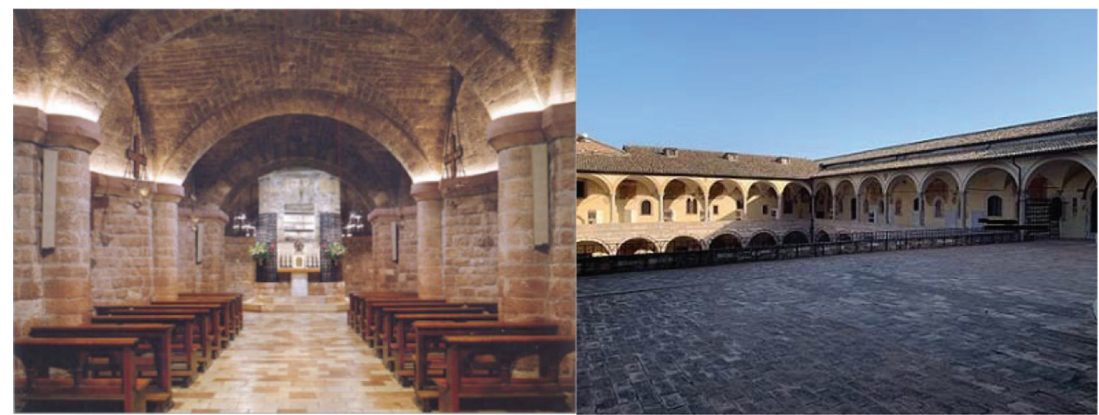

(a)

(b)

Figure 3: View of the: (a) Tomb of Saint Francis; (b) cloister.

a library and enough space for hosting spiritual and cultural activities. Unique and complex cultural heritage sites, such as this, require a significant effort to ensure visitor security and safety, in ordinary condition and during mass events.

In Figs. 2 and 3, some pictures of significant parts of the site are shown.

\section{DESCRIPTION AND ANALYSIS OF THE SITE FROM THE CROWD EVACUATION POINT OF VIEW}

The purposes of the work are:

1. A preliminary analysis of the characteristic factors that affect the level of risk.

2. The identification of a set of emergency scenarios to be modelled in order to identify the critical issues that can affect a safe evacuation of people.

For this reason, it is important to analyse the site properly from the crowd evacuation point of view.

\subsection{Description of the site, internal connections and visiting paths}

Figures 4 and 5 show the layout of the entire Basilica, divided into the four floors that compose it. Floor 0 represents the Tomb, floor 1 represents the lower Church, floor 2 represents the upper part of the cloister and floor 3 represents the upper Church.

The two Churches are connected by a staircase, just over $1 \mathrm{~m}$ wide, located in the left and right transept of the lower Church. From here it is possible to go up to the upper part of the 


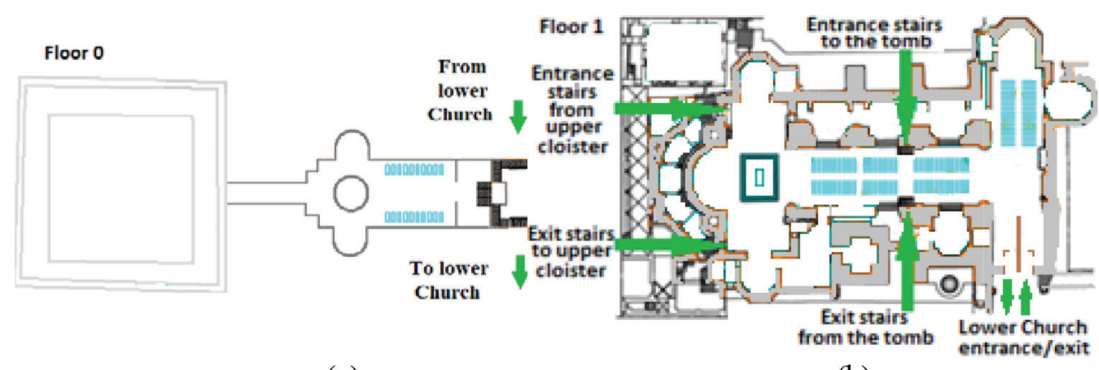

(a)

(b)

Figure 4: Layout of the: (a) Tomb; (b) lower Church.

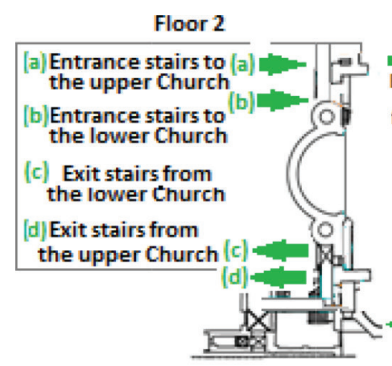

(a)

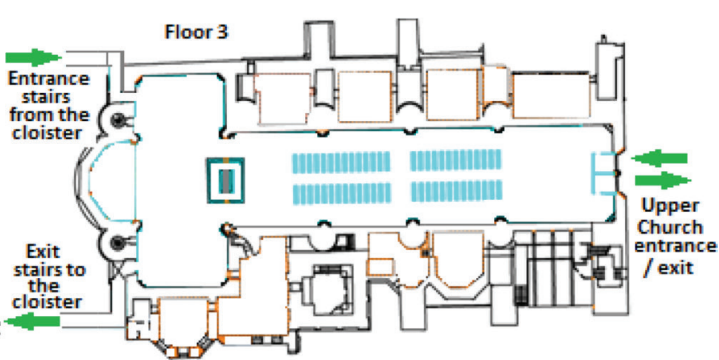

(b)

Figure 5: Layout of the: (a) upper part of the cloister; (b) upper Church.

cloister and from here, through another staircase about $1.5 \mathrm{~m}$ wide, it is possible to reach the upper Church. A similar and symmetrically located path allows to reach the lower Church starting from the upper Church.

The access to the Tomb, which is a limited and very much visited area, is guaranteed by the stairs located about halfway along the central nave of the lower Church. Particular attention must be paid to the geometry of this connection. The entrance/exit stairs, one for descent (on the right) and one for the ascent to the lower Church (on the left), both $1.50 \mathrm{~m}$ wide, lead to a unique bidirectional scale about $3.50 \mathrm{~m}$ wide, as shown in Fig. 6.

There are two entry / exit points for each Church both characterized by separation of flows for access and exit. In particular, the entrance of the lower Basilica has a total width of about $3 \mathrm{~m}$, with a central division for the separation of the flows that extends internally for about $10 \mathrm{~m}$.

The traditional tour starts from the square in front of the lower Church to enter the Basilica. From here it is possible to pass to visit the Tomb, accessed through the stairs located on the sides of the central nave then, going up again in the lower Church, it is possible to reach the upper part of the cloister and after having walked through it is possible to reach the upper Church and then exit outside through the main exit.

It is important to keep in mind that pilgrims and visitors are not always on the move during the visit since they can remain in adoration and prayer seated on the appropriate benches or admire the frescoes that cover the perimeter walls.

The greatest crowding, which could become critical in particular conditions, occurs at points of interest with stationary crowding such as the Tomb and the paintings and in the connections between the different places of the Basilica and the gates. 


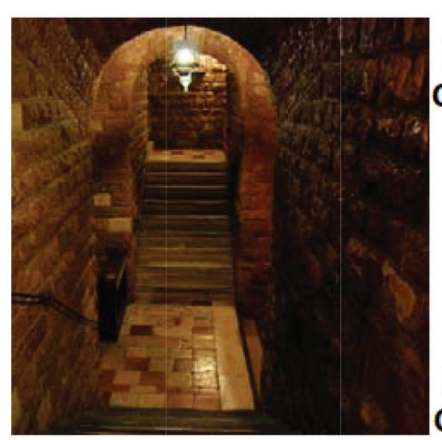

(a)

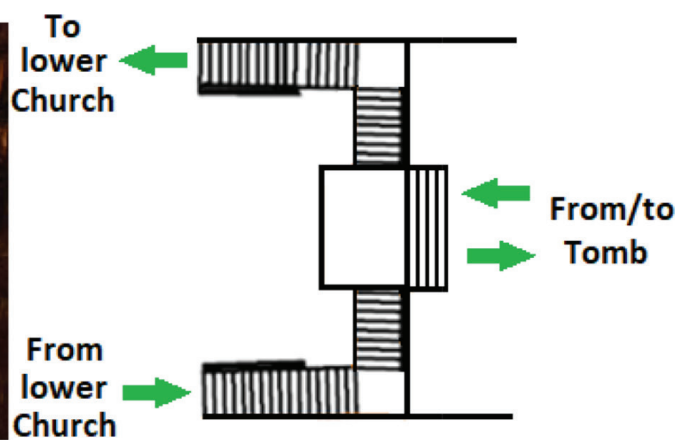

(b)

Figure 6: Entrance/exit stairs of the Tomb: (a) picture; (b) layout.

\subsection{Description of considered event to be analysed}

After describing the site to be analysed, it is necessary to examine the peculiar factors of the considered event attributable, as mentioned above, to site and visitor parameters. The analysis is carried out considering only the visit of the visitors and pilgrims as it represents the main motivation that drives them to Assisi and therefore the scenario that is most likely to occur. For this reason, other specific factors due to concerts, visits of the Pope and other are not considered.

The considered event is of religious kind and it takes place in a well-defined place with clearly defined physical boundaries. Particular attention must be paid to the most crowded accesses and points. Considering that the site is already existing and steady and considering its historical and cultural importance, the structure represents a constraint rather than a design variable.

Due to the kind of place and visitors, it is reasonable to suppose that alcohol and / or drugs, which could encourage dangerous phenomena, are not consumed by pilgrims/visitors so that they are characterized by a relaxed psychological condition.

People mobility is quite variable since visitors/pilgrims can be walking, standing or sitting within the considered site.

On the site children, elderly, pregnant women or people with disabilities can be present so it is necessary to provide special services that meet everyone's needs. In this regard, for example, in the case of elderly people or those with movement difficulties, a reverse visit route is proposed with respect to the traditional one to facilitate the use of downhill stairs, or the use of special lifts.

Due to the kind of site, visitors can also belong to groups (friends, family, organized groups, etc.) and they can be characterized by different age and gender. For the same reason, visitors/pilgrims are also characterized by different nationalities and cultures and this aspect strongly influences their behaviour in term of interpersonal distance and spatial movement. Further, visitors/pilgrims cannot know the site very well and this represent another vital aspect which can strongly influence emergency behaviour.

The key parameter that most affects the level of risk is represented, as with all the crowded events, by the high number of participants and their spatial density.

It is therefore necessary to focus on emergency conditions by analysing the outflow of visitors/pilgrims from the site in order to manage it in a safe way. In this way it is possible avoid of worsening an already possible danger situation, thus avoiding incidents of endogenous nature induced by the crowd. 


\section{CROWD EVACUATION ANALYSIS OF THE CONSIDERED SITE}

Simulation represents a tool that can help professionals to evaluate the performance of the exodus of a complex site with significant gatherings of people, like the one considered, highlighting the possible need of implementing prevention and / or protection measures and to evaluate its effectiveness. In this present work the simulation model of building EXODUS software is applied to analyse crowd evacuation flows. building EXODUS is a part of EXODUS that is a suite of software packages developed by the Fire Safety Engineering Group at the University of Greenwich, UK. EXODUS can be used for both evacuation simulation and pedestrian dynamics/circulation analysis. The software has been developed to meet the challenging demands of performance-based safety codes. It is based on a highly sophisticated set of sub models and it shatters the mould of traditional engineering analysis to produce realistic people-people, people-fire and people-structure interactions. Therefore, it allows to test more designs in less time to reach the optimal solution, free of the high cost and potential danger associated with human evacuation trials. The different software packages that compose EXODUS are tailored to the building, maritime, rail and aircraft environments.

\subsection{Preliminary hypotheses and considerations of simulation analysis}

In the following, the preliminary hypotheses and considerations regarding the simulations carried out are illustrated, to avoid the obtained results of the considered scenarios to be misunderstood.

The developed scenarios are not aimed at investigating the causes that trigger evacuation, but rather the efficiency of the emergency runoff, evaluating the probable behaviour assumed by people in such conditions. In the simulations, the negative effect of the deterioration of the boundary conditions on the movement performance is not considered, as in the case of fire triggering, collapse of the structure or terrorist attacks. Therefore, another assumption is represented by considering invariable the environmental conditions of temperature and of the structure and the absence of toxic gases, assuming the total use of site itself (stairs, doors, paths, etc.).

It is also assumed that pilgrims/visitors react to the danger simultaneously in the various floors: the whole population was given the same range of pre-movement time. Since the site is composed of several levels and the emergency is generally activated at well-defined points, it could represent an interesting future investigation and development of the present work to consider different activation times of people's movement. If, for example, an incidental event begins in the upper Church, people standing in the other floors may have no perception of the danger at the same time with consequent delay in the activation of the exodus. This evaluation, for different accident scenarios, can provide interesting results in order to analyse the impact of people characterized by longer pre-movement times on the total evacuation performance, such as a possible slowdown.

Despite the continuous and numerous studies, gaps related to the prediction of human behaviour in emergency conditions are still existing, especially regarding the quantification of performance in terms of speed of movement and time of pre-movement. This leads to inevitable hypotheses on the values about these parameters. Regarding the pre-movement time, a variable range between 20 and 60 seconds was assumed. So reduced values compared to ordinary fire evaluations values related to buildings are due to the lack of social attachment to the considered site because of the occasional nature of the visit. Unlike homes or workplaces, people do not have objects to be recovered or even particular actions to be terminated 
in the considered situation. Further, it is to consider the actual worldwide context where people are more and more afraid of gathering in large crowds and even just a sudden or loud noise can generate precipitous and dangerous escapes.

No assumptions were made about cultural differences and this may represent a limit of the simulation model since evaluation of different behaviours due to different culture could lead to a difference between the simulated results and the real results.

Due to the considered occasional nature of the visit, people are only familiar with the followed visiting path. Since the ordinary visit route starts from the square in front of the lower Church and since there are no exit signalling systems, it is reasonable to assume that during an emergency exodus most people retrace the route followed from the considered square. In all the simulated scenarios, a probability of knowledge of exit port 2 (related to the square in front of lower Church) equal to 1 was attributed to all people present in the different floors while a lower probability of knowledge (0.3) for exit port 1 (related to the upper Church) was attributed only to people present on that floor.

\subsection{Description of the executed simulations}

The analysis is also aimed at evaluating the effect of family attachment on exodus. In dangerous situations the family groups tend to remain compact and indeed the risk of physical damage is assessed to be less severe than the breakdown of emotional ties. Since the Basilica of Saint Francis in Assisi, unlike workplaces, is a place visited by groups rather than individuals, it seemed appropriate to examine this scenario in order to verify whether the presence of cohesive groups in any way influences the evacuation in terms of total exodus and congestion times. It is to be specified that in this work it is considered that only small groups remain united (of about 4 people): it is assumed that cooperation is null for organized groups formed by many people such as bus trips (it is unlikely that 50 people, that is the number of seats of ordinary bus, can all move together). This assumption is also confirmed by the current research carried out concerning the behaviour of the masses which states that the groups composed by more than 4 people tend to divide into smaller groups. [6-13].

The analysis is also aimed at verifying the influence of overcrowding on evacuation times and on the efficiency of evacuation.

For the purposes described above, two model configurations have been implemented. The first involves the absence of cooperation and communication during evacuation, so each agent (that is, the term used to refer to each individual simulated by the used software) has an isolated and individual behaviour. In this case, everyone moves according to their physical and psychological characteristics. The second configuration instead foresees the presence of a certain percentage of family groups, where each of them behaves in a gregarious manner. The EXODUS Gene parameter of the software was used to represent the individuals belonging to a specific group. Individuals who share, within a radius of $2 \mathrm{~m}$, the same Gene will be part of the same group sharing information about the route and the exit to use. For the purposes of the simulations carried out, it must be specified that the individuals within the group move by adapting their speed to the slowest individual and begin to evacuate following the lowest response time of all those that constitute the group.

For each configuration, more simulation rounds have been executed by varying the number of people present within the structure and, in the case of the second configuration, the percentage of family groups. 
Specifically, 6 different scenarios were considered:

1. Scenario 1: considers the first configuration of the model, assuming the individuality of the exodus and a number of individuals equal to 1785 .

2. Scenario 2: considers the first configuration of the model with an increase equal to $25 \%$ of individuals with respect to scenario 1 , that is 2232 individuals.

3. Scenario 3: considers the first configuration of the model with an increase equal to $25 \%$ of individuals with respect to scenario 2 , that is 2790 individuals.

4. Scenario 4: considers the second configuration of the model, assuming that $30 \%$ of the total people (1785) belong to family groups. Therefore, 139 groups of 4 people were formed with a total of 1229 individuals $(1785-(139 \times 4))$.

5. Scenario 5: considers the second configuration of the model with an increase equal to $25 \%$ of individuals with respect to scenario 4 , that is 2232 individuals. It is assumed that this increase is represented only by families, which varies from $30 \%$ to $44 \%$ of the total. 107 groups were then added and 19 individuals to reach the total number of people of the considered scenario (the distribution of people between the scenarios in the first and second configuration is the same). Totally, there are 246 groups (984 people) and 1248 individuals.

6. Scenario 6: considers the second configuration of the model with an increase equal to $25 \%$ of individuals with respect to scenario 5, that is 2790 individuals. About $50 \%$ of individuals is represented by groups, ranging from $44 \%$ to $46 \%$ on the total. In fact, 72 groups (288 people) and 270 individuals were added. Totally, scenario 6 considers 318 groups (1272 people) and 1518 individuals.

Since the buildingEXODUS model is a probabilistic model, more simulations have been carried out to obtain more precise and valid results of the implemented model.

\subsection{Simulations parameters}

The power of the used software relies on the possibility of inserting and characterizing the expected population by means of a series of variables, associating them with each individual to consider their heterogeneity, then basing the movement on these characteristics.

As already written above, Assisi attracts different pilgrims/visitors whose specific gender and age group cannot be specified, as for a rock concert where a mostly young population is expected. For this reason, for the realization of the simulation model, four main groups, or populations, have been created. They are composed of young men / women aged between 17 and 45 and adult men / women aged between 45 and 80 years, with a uniform distribution of $25 \%$ each. Each population has been assigned values or ranges of values for all the parameters that buildingEXODUS considers for the random determination of individual characteristics. It is important to remember that gender and age affect the physical and movement skills of individuals. An important consideration concerns the speed entered: the lack of scientific data relating to real emergency scenarios leads to assuming only hypothetical velocity values that can give rise to approximations of reality. This factor could represent a possible limitation of the considered model.

The distribution of pilgrims on the various floors of the Basilica can certainly influence the results of the simulations carried out for the difference of speed and flow between the floors and the stairs. For example, by distributing the most of individuals in the main exit floor 
(lower Church), relatively larger congestions can be expected on the door of the lower Church and lesser congestions on the stairs, with respect to a situation where the most of individuals are on the second floor, with an equal crowding.

For the simulations carried out in this work a quite uniform distribution of individuals has been assumed between the various floors of the considered site. Different results can be obtained by distributing the individuals in a different way within the Basilica.

Further, regarding scenarios 1 and 4 (crowding equal to 1785 people) the individuals have been initially placed at points of interest with stationary crowding, as follows:

1. 441 individuals are placed in the upper Church, assuming that all the benches and seats are occupied and distributing the rest of individuals along the sides of the Church close to the paintings.

2. 372 standing individuals are placed in the upper part of the cloister.

3 . in the lower Basilica it is assumed that the benches and seats of the central nave are completely occupied (265 individuals) and that the remaining individuals are positioned along the side walls near the paintings $(137+131+101)$. Some people $(34)$ are also causally arranged in a lateral Chapel (the Chapel of St. Catherine).

4. 304 individuals are placed in the Tomb (that represents a very crowded situation).

It should be noted that individuals and / or groups have not been included in the square in front of the lower Church, although the crowding could be considerable there. The choice is justified by the purpose and modality of the simulations, to assess the influence of the groups on total evacuation. The presence of people in that area, especially near the exit door of the lower Church, certainly influences the exodus in a negative way. For the scenarios that foresee the population increase $(2,3,5,6)$, the percentage increase was applied to each floor so that a proper comparison of the different crowding situations can be done (the ratio between the individuals in the various floors in the different scenarios does not change).

\subsection{Results}

In the following, the stochastic nature of the decision-making process employed by the model requires the execution of a great number of simulations with the same input data. In this work, 100 simulations were performed for each scenario. Each simulation returns for each individual the significant parameters chosen as output and the average of the values obtained for each of them. Anyway, the differences in the overall results are of a few seconds, since the agents and nodes adopted between the various simulations have not been varied. The changes are only related to the randomness by means of which the simulation model associates the agent with the node: an individual can be placed at a certain node in a certain simulation and at another node in another simulation. To obtain results characterized by significant differences, it is possible to change the nodes occupied and / or the characteristics of the agents.

The analysis is based on the average results obtained from the 100 simulations. Since the results are likely to follow a certain distribution, for example Gaussian, and therefore each observation can fall anywhere on the curve, for a specific scenario and configuration it is possible to determine a range of evacuation performances that is likely to be obtained. Therefore, statements like 'the evacuation time for this scenario is 1000 seconds' cannot be done. What can be achieved is an understanding of how the structure / population / environment considered together as a whole system can behave in the presence of a set of predefined conditions. 
The parameters which are considered significant for the purposes of the work are represented by:

1. TET (total exodus time): time that all the people take to reach a safe place, starting from the beginning of the simulation and therefore including the pre-movement time.

2. PET (personal exodus time): time that each individual takes to reach a safe place, including the pre-movement time.

3. CWT (cumulative waiting time): total time that each individual spends stationary waiting to resume movement.

Building EXODUS uses these last two parameters (CWT and PET) to define personal evacuation efficiency (PEE). PEE is calculated based on the equation 1-CWT / PET. The agents that do not remain waiting in a stationary position, which are therefore characterized by a null CWT, are characterized by a PET equal to 1 , indicating that their evacuation is optimal and efficient. The greater the time interval spent on congestion, the lower the efficiency of evacuation.

Although the model uses this parameter to indicate the efficiency of evacuation, it should be noted that it cannot represent the efficiency of the safety system. From this point of view, in fact, efficiency can be defined as the ability of the system to reach the desired goal (exodus of people) with the least use of resources (in the shortest possible time). It must therefore be a function of the total time of exodus and of the considered population. With the same number of individuals, the system that can evacuate everyone in the shortest time is more efficient.

Based on the hypotheses and considerations illustrated above, the results of the total exodus time obtained as the average of the values of the 100 simulations are shown in Fig. 7.

Figure 7 allows us to obtain important information related to the influence of the creation of family groups and crowding on the performance of the evacuation.

Against expectations, the total time of exodus is smaller, albeit slightly, in the case of the second configuration of the model which considers the family attachment (orange histogram) compared to the first which assumes instead an isolated and individual behaviour of people (blue histogram). This could be justified considering that in the simulations with the family groups the time of pre-activation of the movement is on average smaller than the simulations with the crowd made up of only individuals, owing this fact to how the simulator forms and makes the groups move. Since the total evacuation time is inclusive of the pre-movement time, it is correct to obtain this result. Further, due to the way the people are distributed on the site and due to considering almost instantaneous response times, queuing areas are formed

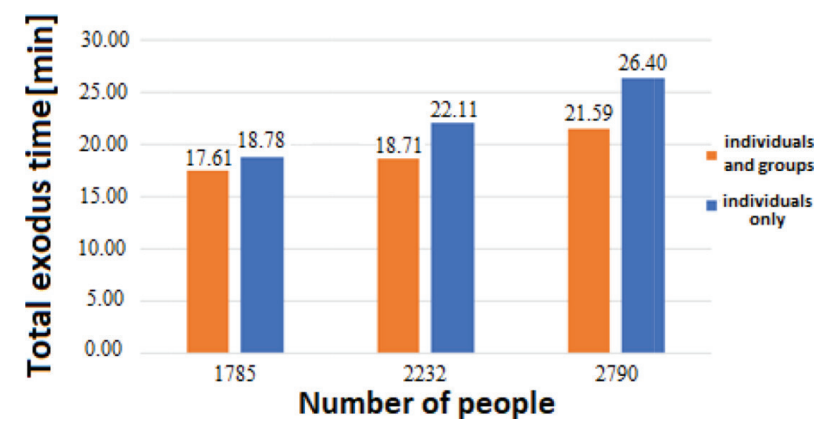

Figure 7: Total exodus time for each considered scenario. 
near the stairs and narrow passages in both configurations. In these cases, the desire of people to stay together or not does not have much influence, probably leading to little differences between the two different simulation configurations.

It should also be remembered that in the various scenarios the flow toward the outputs is different: in the scenarios with the family groups $(4,5,6)$ there are more agents that use the passage on the floor of the upper Church compared to scenarios 1,2,3. This could be caused by the presence of groups. While individuals choose individually the path to follow, the group of 4 people moves to the same safe place presumably leading to greater use of the door. Considering that the total time of exodus represents the personal time of evacuation of the last individual and that reasonably this is the farthest from the exit door, if the flow through the upper floor is increased, the number of people that use the door of the lower Church decreases, with the consequent decrease of the total time of exodus.

Regarding the influence of crowding on evacuation performance, from Fig. 7 it is possible to deduce that, for both configurations, the increase of people leads to an increase of the total exodus times, in accordance with what one could intuitively expect.

Although the total evacuation time represents a useful parameter, it does not represent the history of the simulation. It is therefore suitable to consider other parameters which provide more information useful to study the phenomenon, which are represented by the effect of family aggregation on evacuation performance. For this reason, the indicator of congestion and queuing phenomena and the cumulative waiting time are properly considered in the following.

The average CWT values were examined for all the 100 simulations carried out and shown in Fig. 8, since they provide useful information regarding the general trend among the various scenarios.

From Fig. 8, it is possible to see that the increase in the number of people has negative effects on CWT. This result agrees with the experimental data: the greater the number of members of the moving crowd, the greater the reciprocal interactions, leading to a less stable and more congested flow with consequent creation of queuing phenomena.

Another interesting result derives from the comparison between the two configurations of the model (individuals and groups or individuals only). In fact, the cumulative waiting time is greater for the second configuration (orange histogram) with respect to the first (blue histogram), as one could intuitively expect. The fact that individuals tend to move in a gregarious way, assuming the same characteristics of movement, leads to the formation of queuing areas and therefore to longer stationary waiting times. However, the related obtained results differ slightly from the quantitative point of view for the two configurations and for the three

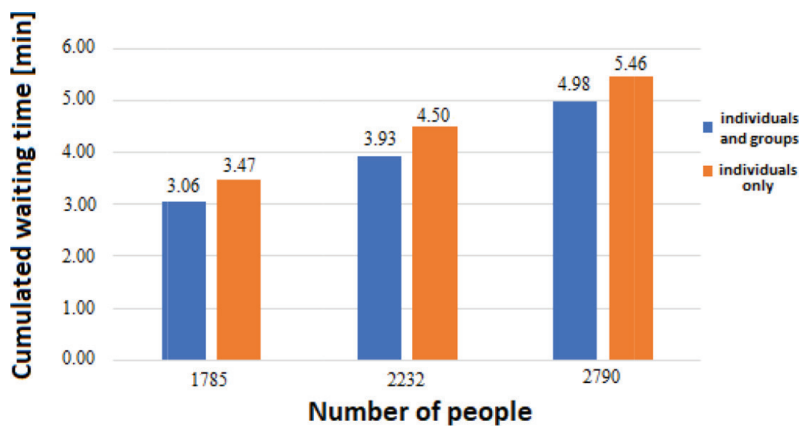

Figure 8: Cumulated waiting time for each considered scenario. 
considered crowding situations. This could be due to the level of congestion that is created near the narrow passages even in the absence of groups, due to the hypothesis of short pre-movement times and the simultaneous evacuation of people on the various floors. For this reason, the CWT values were analysed for more crowded and therefore more critical scenarios. As an example, the distribution of people as a function of cumulative exodus time, for a single simulation, is shown in Fig. 9. It is anyway been verified that the shown values do not change significantly between the various simulations.

From Fig. 9 it is possible to see that in both configurations there is a percentage of individuals characterized by waiting times exceeding 8 minutes and that this percentage is greater for simulation with individuals and groups than that with individuals only, indicating a higher level of queuing.

However, although on average a greater cumulative waiting time was obtained for simulations with individual and groups, analysing the individual values, higher peaks are revealed for the configuration that considers the crowd formed by individuals only, according with what is assumed for the previous illustrated results about the total time of exodus. The high values of the queue, in fact, can reasonably be due to the most distant individuals who, having to cross three floors and the related stairs, are subjected to queuing in narrow passages, with longer waits. Since the number of individuals moving through longer distances decreases in simulations with groups, it may be reasonable to expect lower peak values in the latter case. It should be noted that a good percentage of the people, greater in the case of all individuals, seems to be able to evacuate optimally, being characterized by waiting times of less than 1 minute. This also explains the relatively low average values obtained from the 100 simulations.

The simulations show that stationary waiting time values exceeding the critical time of 8 minutes are reached, beyond which the probability that spontaneous panic phenomena are generated is high. It is therefore necessary to design prevention and / or protection measures aimed at reducing the likelihood of a mass disaster, to avoid the behaviour of the crowd leading to situations that slow evacuation since the psycho-physical behaviour represents a vital factor for a safe emergency runoff. Due to the nature of the site, it is not possible to implement structural changes, such as the design of new gates, the enlargement of the existing one or of the stairs. For this reasons the solutions that can be adopted are related to the design of an acoustic and luminous signalling system, even supported by a proper IoT/IoE system [1-3] (such as the one that is going to be realized), that leads people through proper smart

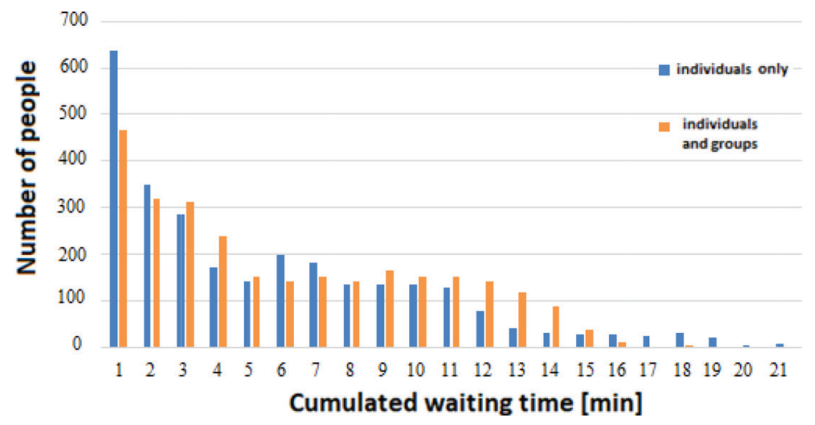

Figure 9: Distribution of the people as a function of cumulative exodus time for a single simulation. 
exodus, to use simply, as an example, also the door of the upper Church to reduce the flow on the stairs and the passage currently used, with a probable improvement on the time of exodus and congestion.

Another solution that can be implemented is represented by the management of the outflow near the exit of the lower Church. In fact, the behaviour of the crowd, highlights that an adequately designed system of barriers can lead to an improvement in the flow of the crowd itself [6-13]. The geometry of the existing gate is represented by an internal barrier, which extends for about $10 \mathrm{~m}$ with the objective of separating incoming and outgoing flows. This division, optimal for the purpose, can be modified to improve the emergency exodus. In these conditions, it is reasonable to suppose that both gates are used for the exodus. However, the results of the simulations in that area reveal that most people use the same passage, represented by the one which minimizes the distance to the exit. For this reason, other simulations have been carried out by modifying the length of the internal barrier extending it properly and executing further simulations with an increase of $1 \mathrm{~m}$. The aim is to verify if a more equitable distribution of the crowd between the two passages can improve the outflow in emergency, above all with reference to the areas characterized by a higher density. The simulations were carried out on the most crowded scenarios where higher congestion values were obtained (therefore on scenarios 3 and 6) and results are shown in Fig. 10.

Figure 10 shows the crowding density at the same moment (11th minute) of the simulation, considering individual and groups, in the actual situation and with the proposed implementation. Red colour represents the area where there is the maximum the density (therefore equal to 4 persons $/ \mathrm{m}^{2}$ ), while the other zones characterized by different colours identify decreasing density (magenta, yellow, green, light blue and blue). From Fig. 10 it is possible to see, from a qualitative point of view, an effective improvement in the distribution of the crowd with the extension of the 3-m barrier to about $4 \mathrm{~m}$ and a fairly even distribution of the crowd between the two passages. Figure 11 refers to the same situation as in Fig. 10 but it considers individuals only.

Therefore, the lengthening of the internal partition seems to lower the level of congestion at the exit passages, thus reducing the probability of a mass disaster induced by the crowd in emergency conditions. It should be emphasized that this preventive crowd management measure guarantees the result obtained since the lengthening of the partition makes the path less used by people in the actual situation as attractive as the currently most used route. Obviously, it is not possible to state that the partition improves the runoff, but it is possible to

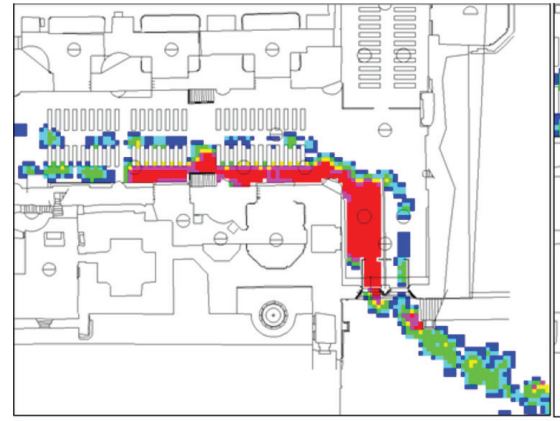

(a)

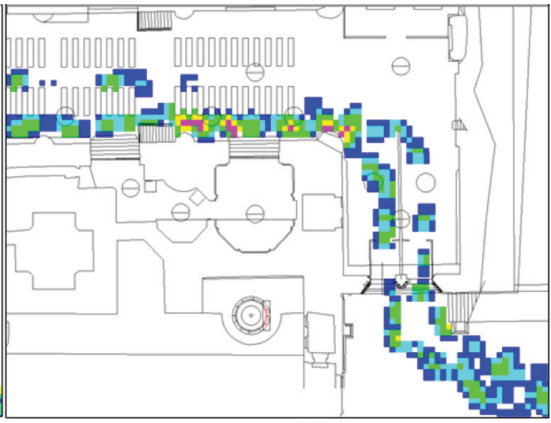

(b)

Figure 10: Density level of people at the 11th minute of simulation for the most crowded scenario (2790 people) considering individuals and groups for: (a) actual situation; (b) with the proposed implementation. 


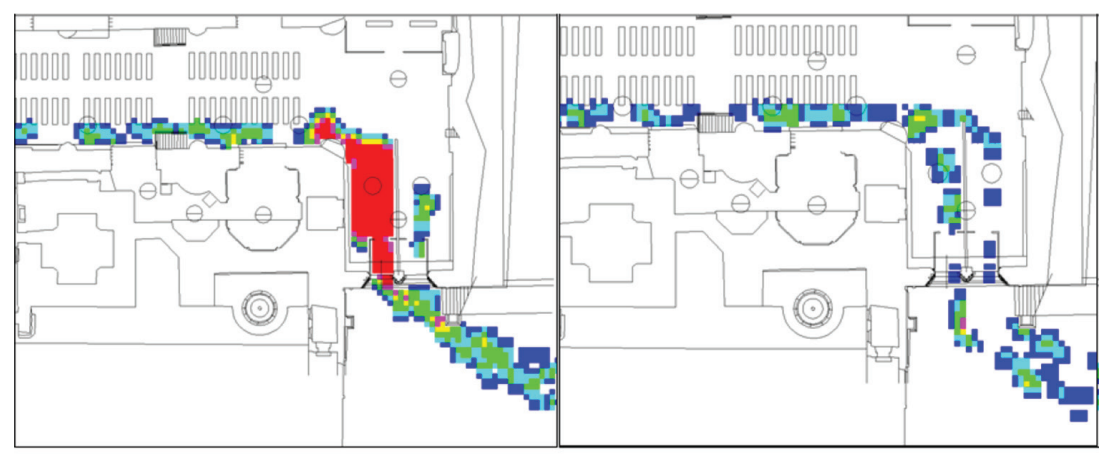

(a)

(b)

Figure 11: Density level of people at the 11th minute of simulation for the most crowded scenario (2790 people) considering individuals only for: (a) actual situation; (b) with the proposed implementation.

deduce that a more equitable distribution of the crowd between the two passages can improve the flow, making the crowd free to move safely.

The results illustrated above in a qualitative way have been properly verified from the quantitative point of view and illustrated in Fig. 12, where the distribution of the cumulated waiting time (CWT) between actual situation and the implementation of the change for scenarios 3 (2790 pilgrims and all individuals) and 6 (2790 pilgrims and partly family groups) are shown.

An effective improvement can be observed for both comparisons shown in Fig. 12. In particular, for scenario 6 it is possible to see that the change leads to a decrease in the number of people waiting in queue overall for a time exceeding 8 minutes which represent a critical threshold. Furthermore, there seems to be a lowering of peak values of about two minutes. Results related to scenario 3 show that even in this case the number of people with a cumulative waiting time greater than 8 minutes decreases. However, the distribution of the tail remains unchanged, with a slight improvement of the peak value which seems to decrease by about 1 minute. The absence of differences for the tail values is an indication of the level of congestion near the stairs and it is reasonable for it to remain constant. Finally, it should be noted that, despite the better outflow, there are no significant differences in the total exodus time.

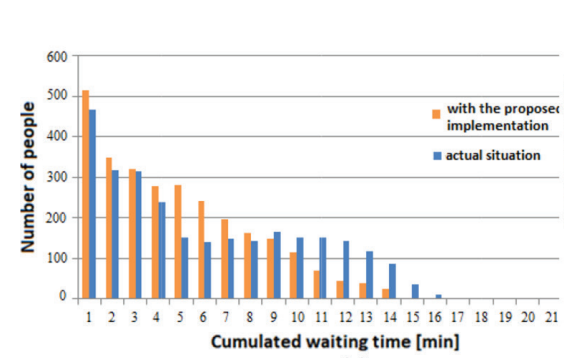

(a)

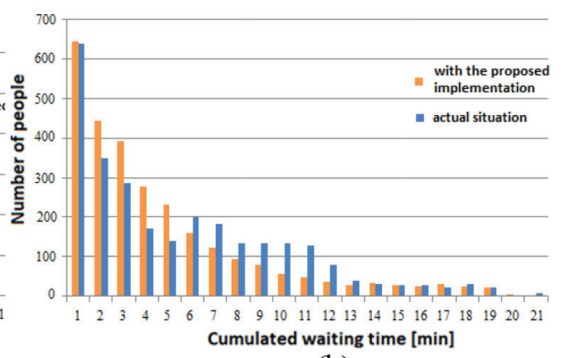

(b)

Figure 12: Distribution of people vs. cumulative exodus time, in the actual situation and with the proposed implementation, for: (a) scenario 6; (b) scenario 3. 


\section{SUMMARY OF THE OBTAINED RESULTS}

The simulations were executed based on different hypotheses and considerations which simplify the real context. The obtained results must therefore be contextualised to the simulated situation and, more importantly, they must be correctly considered, since the forecast of evacuation performance by means of simulation models represents a useful support to evaluate the exodus performance from a given site and to know the way the parameters related to the behaviour of individuals influence the evacuation.

In particular, the simulations executed in this work show how the family attachment that drives individuals to move in groups influences the performance of the evacuation. It has been observed that this gregarious behaviour leads to higher levels of congestion compared to considering the crowd formed only by individuals who move according to their own characteristics.

Furthermore, it has been verified that crowding affects the performance of the exodus in terms of total evacuation time and queuing levels. It is necessary to note that despite the relatively low crowding density which has been considered, run-off times are quite high. This could depend on the people that use, almost all of them in the considered situation, a single passage represented by the one more familiar for them.

Finally, a part the use of a proper IoT/IoE system for a smart management and evacuation (such as the one which is going to be realized), the possibility of modifying the exit of the lower Basilica in order to improve congestion conditions in that area was evaluated. The obtained results show that a more equitable distribution of the crowd on the two exodus passages reduces the level of congestion and therefore the probability of a mass disaster induced by the crowd. Improving the distribution of people makes the outflow more orderly and less dangerous.

It is necessary to remember that the analysis is carried out considering only the visit of the visitors and pilgrims as it represents the main motivation that drives them to Assisi and therefore the scenario that is most likely to occur. Further, due to the kind of place and visitors, it has been supposed that alcohol and/or drugs, which could encourage dangerous phenomena, are not consumed by pilgrims/visitors so that they are characterized by a relaxed psychological condition. Another assumption is represented by considering invariable the environmental conditions of temperature and of the structure and the absence of toxic gases, assuming the total use of site itself (stairs, doors, paths, etc.). Further, it is also assumed that people react to the danger simultaneously in the various floors that is the whole population was given the same range of pre-movement time variable between 20 and 60 seconds; no assumptions were made about cultural differences; occasional visitors, that is people which is only familiar with the followed visiting path, are considered.

Further developments of the present work could be represented by not considering the various conditions used for the simulations carried out, which are indicated above.

\section{CONCLUSIONS}

The crowd evacuation analysis of the Papal Basilica of Saint Francis in Assisi that, together with the Sacred Convent, represent a unique and specific cultural heritage site visited by millions of pilgrims and visitors from all over the world each year, was performed.

To reach the desired goal, a proper simulation model of people evacuation was set up, considering the fundamental aspects of interaction between the architecture and layout of the Basilica and the behaviour of the crowd.

The obtained results provide a series of important information for people evacuation management that lead to a more regular exit flow with a decrease in the time of stationary waiting 
and, therefore, with a decrease of the probability of generating spontaneous panic phenomena induced by the crowd.

This work represents only a basic study that can be further developed to obtain more useful results for the desired purpose.

\section{ACKNOWLEDGEMENTS}

The authors wish to thank Cristina Ceccarelli for her contribution in the study development.

\section{REFERENCES}

[1] Garzia, F., Sammarco, E. \& Cusani, R., The integrated security system of the Vatican City State. International Journal of Safety and Security Engineering, 1(1), pp. 1-17, 2011. https://doi.org/10.2495/safe-v1-n1-1-17

[2] Gambetti, M., Garzia, F., Vargas Bonilla, F. J., Ciarlariello, D., Ferrer, M. A., Fusetti, S., Lombardi, M., Ramalingam, S., Ramasamy, M., Sacerdoti, S., Sdringola, A., Thirupati, D. \& Faundez Zanuy, M., The new communication network for an Internet of Everything based security / safety / general management / visitor's visitors' services for the Papal Basilica and Sacred Convent of Saint Francis in Assisi, Italy. Proc. of IEEE International Carnahan Conference on Security Technologies, Madrid (Spain), 2017.

[3] Garzia, F., Implementing an Internet of everything system in the archaeological area of Quintili's Villa in the ancient Appia route park in Rome. WIT Transactions on the Built Environment, 174, pp. 261-272, https://doi.org/10.2495/safe170241

[4] Garzia, F. \& Lombardi, M., Safety and security management through an integrated multidisciplinary model and related integrated technological framework. WIT Transactions on The Built Environment, 174, pp. 285-296, https://doi.org/10.2495/safe170261

[5] Guarascio, M., Lombardi, M., Rossi, G. \& Sciarra, G., Risk analysis and acceptability criteria. WIT Transactions on the Built Environment, 94, pp. 131-138, 2007.

[6] Borghini, F., Garzia, F., Borghini, A. \& Borghini, G., The Psychology of Security, Emergency and Risk, WIT Press, 2016.

[7] Sime, J.D., Crowd psychology and engineering. Safety Science, 21(1), pp. 1-14, 1995. https://doi.org/10.1016/0925-7535(96)81011-3

[8] Martella, C., Li, J., Conrado, C. \& Vermeeren, A., On current crowd management practices and the need for increased situation awareness, prediction, and intervention. Safety Science, 91, pp. 381-393, 2017. https://doi.org/10.1016/j.ssci.2016.09.006

[9] Appert-Rolland, C., Cividini, J., Hilhorst, H.J. \& Degond, P., Pedestrian flows: From individuals to crowds. Transp. Res. Procedia, 2, pp. 468-476, 2014. https://doi. org/10.1016/j.trpro.2014.09.062

[10] Campanella, M., Hoogendoorn, S. \& Daamen, W., Effects of Heterogeneity on SelfOrganized Pedestrian Flows. Transportation Research Record: Journal of the Transportation Research Board, 2124(1), pp. 148-156, 2009. https://doi.org/10.3141/2124-14

[11] Helbing, D., Johansson, A. \& Al-Abideen, H.Z., Dynamics of crowd disasters: An empirical study. Physical Review E - Stat. Nonlinear, Soft Matter Phys., 75(4), pp. 1-7, 2007. https://doi.org/10.1103/physreve.75.046109

[12] Löhner, R., On the modeling of pedestrian motion. Applied Mathematical Modelling, 34(2), pp. 366-382, 2010. https://doi.org/10.1016/j.apm.2009.04.017

[13] Moussaïd, M., Perozo, N., Garnier, S., Helbing, D. \& Theraulaz, G., The walking behaviour of pedestrian social groups and its impact on crowd dynamics. PLoS One, 5(4), pp. 1-7, 2010. https://doi.org/10.1371/journal.pone.0010047 